\title{
Integration of Process Planning and Scheduling using Resource Elements
}

\author{
J.D.A. Carvalho and N.N.Z. Gindy \\ Department of Manufacturing Engineering and Operations \\ Management, University of Nottingham, Nottingham NG7 2RD, \\ United Kingdom, Tel +44-115-9514031, Fax +44-115-9514000, \\ Emailepxjdc@unicron.nott.ac.uk
}

\begin{abstract}
This paper outlines a new approach utilising generic capability units termed 'resource elements' (REs). REs describe the capabilities of machine tools and machining facilities, and represent the process planning information. A prototype integrated environment for process planning and scheduling in which REs are used for both the generation of nonlinear process plans and production scheduling is described. It is shown to offer significant improvements in manufacturing system performance and REs are seen to provide a powerful and practical approach to integration.
\end{abstract}

Keywords

Process planning, scheduling, integration, resource elements, nonlinear process plans

\section{INTRODUCTION}

Manufacturing companies are divided into specialist departments performing computer aided design (CAD), computer aided process planning (CAPP) and production planning and control (PPC). These activities are normally performed sequentially by departments, with most performanceimproving effort directed towards refining and perfecting individual functions rather than performance across functions.

Today, CAPP and scheduling remain essentially separate activities. Process planning is considered a time-independent activity that generates an optimised set of sequenced operations and resources for transforming part design into a finished product, while scheduling is treated as a time-dependent function aimed at the utilisation of resources to satisfy the process plans for a number of products (Chryssolouris and Chang 1985, Kuhnle 1990).

Difficulties in integrating CAPP and PCC relate either to the functionality of these systems or to their use of data. While process planning systems focus on the operations to be performed on single parts, scheduling systems deal with both multiple parts and multiple products to be manufactured within the same system. Conventional scheduling systems also adopt a global view and thus aim to 
optimise machine utilisation and maximise throughput to meet production targets. Schedulers can normally only handle linear sequential process plans and resources are often combined into capacity groups for the purpose of load balancing (ElMaraghy 1993).

Production disruptions and bottlenecks occur on the shop floor due to resource shortages and routes have to be changed to suit demand; however, this is done without full consideration of its implications for the overall operation of the shop floor. The lack of communication between process planning and scheduling therefore leads to higher costs and is a serious obstacle to achieving effective integrated manufacturing systems.

Many shortcomings in current CAPP and scheduling industrial practices are the result of several assumptions: (i) Process planning is a static activity, influenced only by component design and its technological constraints, and thus focuses on technical manufacturing analysis of products to satisfy their design intent. (ii) Scheduling is strictly sequential, to be performed only after process planning, and therefore focuses solely on capacity constraints and efficient resource time-allocation. (iii) There is unconstrained resource availability during component manufacture. Moreover, process planning is performed such that some 'desirable' routing normally utilising the most capable machine tools is repeatedly selected for each component.

In modern, highly dynamic, responsive industrial environments, none of the above assumptions is realistic or applicable. Process planning and scheduling may have conflicting goals, but a significant potential for improvement in the $\mathrm{CAD} / \mathrm{CAM}$ information integration can be achieved if the gap between CAPP and scheduling can be bridged.

There are three basic approaches towards integration of CAPP and PPC (EIMaraghy 1993). The global integration approached is based upon high level integration of functions and CIM modules. Each CIM module (CAD, CAPP, MRPII) maintains its own database and a global update scheme is devised to achieve information integration. This method is data-intensive and does not address the need for nonlinear plan representation (ElMaraghy and ElMaraghy 1993).

The second approach attempts unification, merging CAPP and PPC into one system with a common structure. Petri-nets are commonly used to model logical and temporal relationships between system entities. The issues of realtime events and related feedback from PPC to CAPP in response to shop floor 'disturbances' are not fully considered, e.g. FLEXPLAN-ESPRIT Project 2457 (Toenshoff et al. 1989, Toenshoff and Detand 1990). Extensions to the approach are made in the new EPRIT sponsored project COMPLAN (Kruth et al 1994) to develop an integrated system for concurrent process planning, scheduling and shop floor control based on nonlinear process plans.

The third approach to integration can be considered an intermediate solution between the first two approaches (ElMaraghy 1993, ElMaraghy and ElMaraghy 1993). This approach is essentially modular where CAPP and PCC are not treated as one system. However, the CAPP and PPC systems need to have the ability to interact with the shop floor disturbances, nonlinear process plans, and dynamic resources and constraints. In this approach, integration schemes utilising a separate module called the 'integrator' were developed to bridge the functional and data gaps between CAPP and PPC (Jack and ElMaraghy 1992).

Real improvement in production control can only be achieved by integration of a CAPP system and a scheduling system that fully utilises the benefits of manufacturing alternatives provided by nonlinear process plans. The actual utilisation of machines is significantly determined early on in process planning and to meet the flexibility requirements of workshop control, the process plan itself has to be more flexible. It is recognised that CAPP and PPC systems need to be able to generate and 
utilise nonlinear process plans, interact with the shop floor disturbances, and dynamically take into account resource availability constraints on PPC (Jack and ElMaraghy 1992).

It is the contention of the authors that one of the main directions for improving manufacturing performance lies in improved interaction and integration between process planning and scheduling. This will lead to a dynamic manufacturing environment capable of reacting to real factory conditions, and will have a significant impact on reducing cost, lead time and inventory as well as improving manufacturing responsiveness.

The reported work is based upon utilising generic (machine-independent) capability units termed 'Resource Elements' both to represent component process planning information and to act as the basis for the generating the production schedule. An integrated environment for process planning and scheduling is currently under development, and is described here.

\section{SYSTEM DESCRIPTION}

The input to the integrated system (Figure 1) is a 'manufacturing job' list of the components to be produced within a scheduling period. It includes: component identification, due date and a priority value associated with each component. The list can be sorted according to component priority or earliest due date depending on the desired manufacturing strategy. The way the components are sorted represents the sequence that they are processed through the proposed system. (1) Each component goes through a generic planning module where machine-independent (RE-based) component process plans are generated. (2) Processing and setup times are generated for each process plan. (3) A simulating model is created based upon the process plans, and (4) this model is simulated for different dispatching rules and different shop floor performance measures. The dispatching rule resulting in a better performance indicator is selected. The final component process plans and schedule are those obtained using the selected dispatching rule.

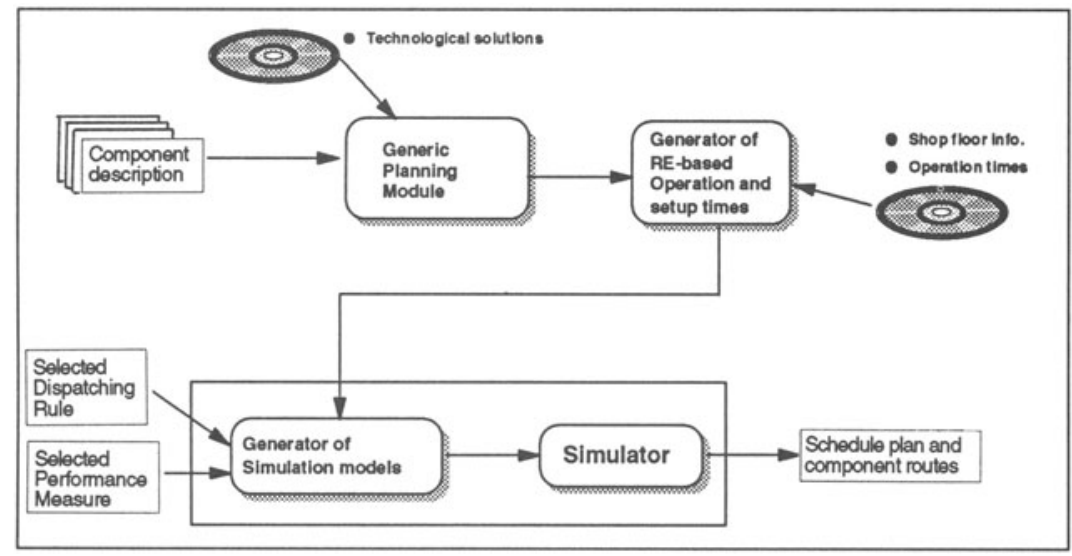

Figure 1 System Overview. 
The results obtained from the simulation module shown in Figure 1 as the output (schedule plan and component routes) are both machine-based although the simulation model is created using REbased information.

The number of components in the list depends on the company's scheduling period. Each manufacturing system has its own minimum limit for the scheduling period representing the time the system needs to check the feasibility of making each component. It depends on the time necessary to prepare and test fixturing devices, tools, NC part programs, to verify resources availability, etc., as well as the frequency that the shop floor's status database is updated. Manufacturing systems that can perform all these tasks rapidly would have very short scheduling periods. Vice versa, long scheduling periods normally imply that the real allocation of jobs to machines will not follow the scheduling plan in order to cope with the unexpected (machine breakdown, resource shortages, etc.), thereby wasting all the effort to 'optimise' the shop floor performance at the planning stage. It is important to have short scheduling periods in order to gain the advantages of the integration.

\subsection{Generic Planning Module (GPM)}

An overview of the GPM is shown in Figure 2. A feature-based component data model is used to represent component information (Gindy and Ratchev 1991). Each component is treated as a set of connected features, with each feature (e.g. hole, slot, step, pocket) relating to a component region which has significance in the context of machining operations. Based on their geometric attributes, features are classified into categories, classes and sub-classes, which may be followed by secondary forms to describe component geometry (Gindy 1989). Technological requirements (dimensions, accuracy and surface finish etc.), which influence the selection of processing methods, are attached to each feature. Features relate to local component regions and alone are unable to describe the structural aspects of component geometry, i.e. the relationships between component features which can influence the selection of its methods of manufacture. Feature relationships are represented using feature connectivity graphs which relate each feature to adjacent features and to the potential processing directions of the component (Gindy et al. 1993).

During generic planning, each component is treated as a set of features that require machining using the available processing system resources. Feature connectivity provide the constraints that may exist on the formation feature clusters that can be machined from a common component direction (potential component setups). Each feature has a multiple set of technological solutions capable of producing the feature geometry and satisfying its technological requirements in terms of accuracy, surface finish, tolerance.

For a machine-independent but machining-facility-specific description of the processing requirements of components, each feasible solution is represented by the necessary REs.

REs can be considered as elementary capability units collectively representing the full capabilities of the machines contained in a machine shop (Gindy et al. 1995). They are machine-shop-specific and describe the distribution (commonality and uniqueness) of the resources among the machine tools contained in a manufacturing cell or machine shop (Figure 3). REs can belong to several machine tools in a manufacturing facility and thus provide a basis for a generic definition of the processing requirements of components. Each component can be represented by a set of unique REs obtained from alternative machine tools without having to specify the actual tools to be used.

During feature level optimisation, the set of REs needed to produce each component feature is determined. An algorithm based upon minimising the variety of resources needed for each 
component is used for optimising the resource set required for individual components. The most appropriate technological solution is then attached to each feature. During resource optimisation the system is capable of selectively avoiding the use of REs which are in heavy demand.

For each component in the manufacturing job list, the output of the GPM is a machineindependent process plan expressed in terms of the REs needed for its execution.

\subsection{Generation of production schedule}

Although the physical entities upon which the production schedule is ultimately generated are machines, component routes are defined generically in the simulation model based upon their RE requirements. The components wait in RE queues for a machine with the required REs to become available. REs act as parallel processing resources thereby increasing the flexibility of utilising system resources. Dispatching rules are used as the basis for simultaneously generating the production schedule and the final process plan for the components in the manufacturing job list.

The discrete simulation package SIMAN (Pegden et al. 1990) generates component routes and schedules and calculates system performance based upon the actual resources used for processing each component in the job list. The system is based on simulation models for capturing the characteristics of the manufacturing system in a mathematical form using a simulation language.

At the start of the simulation, generic plans are created sequentially for the components in the manufacturing job list and the simulation model is executed. The simulation model assumes the use of RE-oriented dispatching rules, to drive the forwards allocation of jobs to machines on a time basis. The system output is a production schedule and the process plans for the components in the job list as well as the system performance indicators.

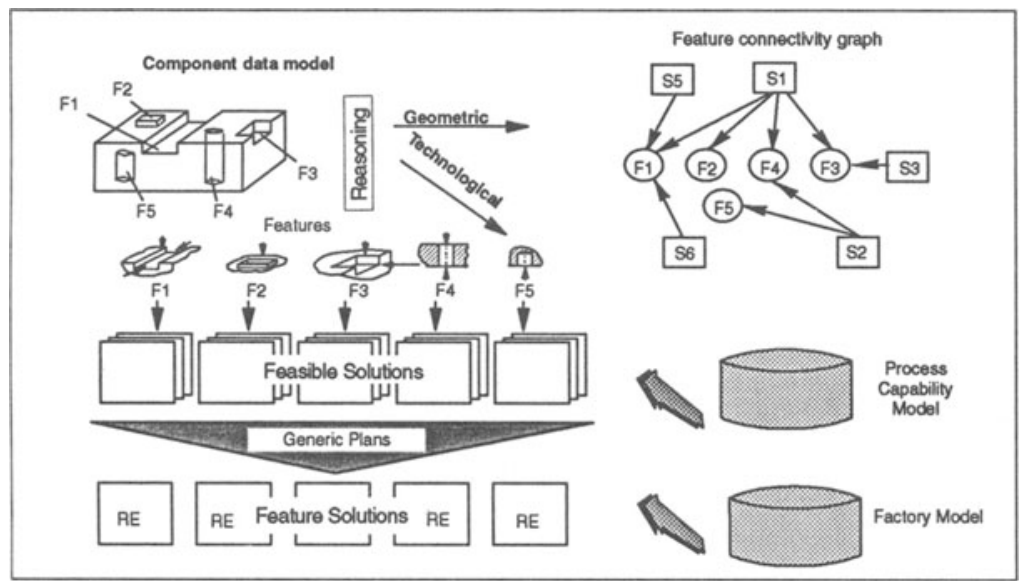

Figure 2 Overview of Generic Planning Module (GPM). 


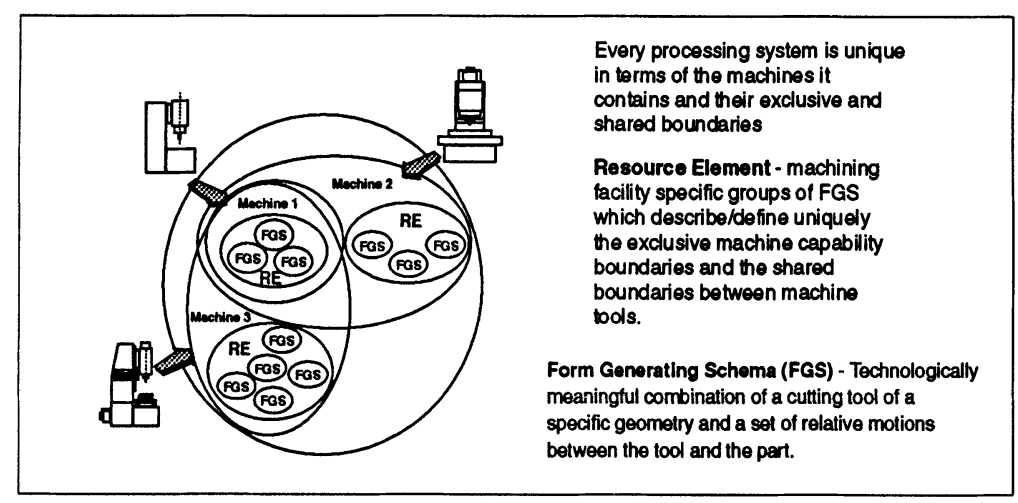

Figure 3 Definition of resource element.

\section{RESULTS}

The results presented here relate to a machining facility containing 22 machine tools operating as a one-of-a-kind production environment. The manufacturing job list within the scheduling period contained 25 components, with 4-12 features per component.

To evaluate the RE approach against the current practice of separately generating component process plans then scheduling whole machine tools assuming infinite resource availability, a production schedule, for the same manufacturing job list, is produced and the results of the two approaches compared.

Three dispatching rules were tested: first in first out (FIFO), shortest processing time (SPT) and earliest due date (EDD). The best values obtained were selected and are presented below. System performance was measured using five performance indicators: average flowtime (AFT), maximum flowtime (MFT), average tardiness (AT), maximum tardiness (MT) and machine utilisation (MU).

\section{DISCUSSION AND CONCLUSIONS}

The machine-based scheduling strategy is based on fixed component routes determined at the planning stage. The only flexibility here is the number of repeated machine tool types available in the machine shop. In the RE-based strategy, on the other hand, REs are used all the way through the process of simultaneously generating component plans and the production schedule for the components in the manufacturing job list.

RE-based integration achieves significant improvements in system performance (Figure 4) since REs offer a sub-machine capability units for use during schedule generation. It therefore increase the choices for optimising the allocation of jobs to available machine tools. Machine-based scheduling, however, has its number of choices limited by the number of the repeated machines and the rigidity of the routes decided at the planning stage. 


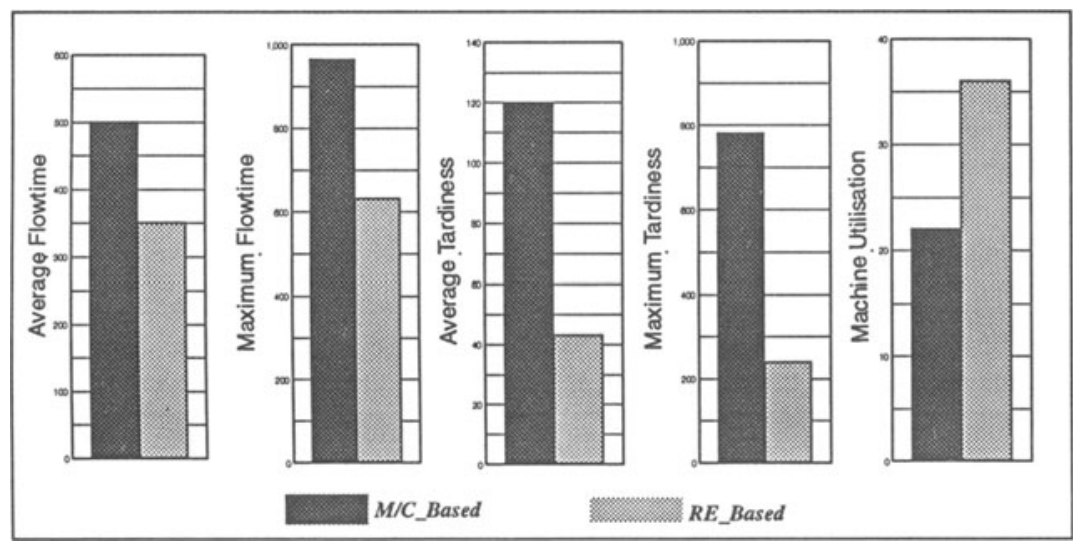

Figure 4 M/C-based strategy versus RE-based strategy.

The case study results show that integrating process planning and scheduling significantly improves manufacturing system performance. The RE concept is shown to be a powerful and practical approach towards this integration and can significantly improve shop floor performance.

The proposed system is a straightforward tool that can easily be applied to manufacturing systems of any size or any level of automation. This system uses the new RE-based way of describing a shop floor which proves to be very efficient. Any existing CAPP and scheduling system can be transformed into a RE-based integrated system, by (i) adding a module between the CAPP system and the shop floor database that transforms the machine-based information into RE-based information; (ii) adapting the scheduling system to schedule machines using RE information; (iii) feeding the scheduling system with the generic plans created by the CAPP system.

\section{AKNOWLEDGMENTS}

J D A Carvalho would like to aknowledge the grant from "Programa Ciencia, BD/2103/92-IB, JNICT, Portugal", under which his part of the work was carried out.

\section{REFERENCES}

Chryssolouris, G. and Chang S. (1985) An integrated approach to integrate process planning and scheduling. Annals of the CIRP, 34(1), 413-417.

ElMaraghy, A. (1993) Evolution and future perspectives of CAPP. Annals of CIRP, 42(2), 739751.

ElMaraghy, H.A. and ElMaraghy, W.H. (1993) Bridging the gap between process planning and production planning and control. Proc. 24th CIRP International Seminar on Manufacturing Systems, June 11-12, 1992. Copenhagen, Denmark, pp. 1-10; also published in Manuf. Sys., 22(1), 5-11. 
Gindy, N.N.Z. (1989) A hierarchical sutructure for form features. Int. J. Prod. Res., 27(12), 20892103.

Gindy, N.N.Z. and Ratchev, T.M. (1991) Product and machine tools data models for computer aided process planning systems, in Computer Applications in Production and Engineering: Integration Aspects (ed. Doumeingts, Browne and Tomljanovich), Elsevier Science Publishers B.V., IFIP.

Gindy, N.N.Z., Huang, X. and Ratchev, T.M. (1993) Feature-based component model for computer-aided process planning systems. Internation Journal of Computer Integrated Manufacturing, 6(1\&2), 20-26.

Gindy, N.N.Z., Ratchev, T.M. and Case, K. (1995) Component grouping for cell formation using resource elements. Int. J. Prod. Res., submitted.

Jack, H. and ElMaraghy, W.H. (1992) A Manual for Interprocess Communication with the MPS (Message Passing System). DAMRL Report No. 92-08011, the University of Western Ontario, London, Ontario.

Kruth, J.P., Detand. J., VanZeir. G., Kempenaers, J. and Pinte, J. (1994) Opportunistic process planning: A knowledge based technique for CAPP applications. Computers in Engineering, Proceedings of the International Conference and Exhibit 94, 1, 227-233

Kuhnle, H. (1990) Prerequisites for CAPP. 22nd CIRP Seminar on Manufacturing Systems, University of Twente, The Netherlands.

Pegden, C.D., Shannon, R.E., Sadowski, R.P. (1990) Introduction to Simulation Using SIMAN. McGraw-Hill, ISBN 0-07-049217-4.

Toenshoff, H.K., Beckendroff, U. and Anders, N. (1989) FLEXPLAN - A Concept for Intelligent Process Planning and Scheduling. CIRP Intr. Workshop on CAPP, Hannover University, Sept. 21-22, pp. 87-106.

Toenshoff, H. K. and Detand, J. (1990) A Process Description Concept for Process Planning, Scheduling, and Job Shop Control. 22nd CIRP Seminar on Manufacturing Systems, University of Twente, The Netherlands.

\section{BIOGRAPHIES}

Jose Carvalho, Lic., Msc, is currently a $\mathrm{PhD}$ student at the Department of Manufacturing Engineering, University of Nottingham, UK. He worked as a lecturer at Universidade do Minho Portugal until 1992. Major interests include CAPP, PPC and CAPP/PPC integration.

Nabil Gindy, Bsc, Msc, $\mathrm{PhD}$, is professor of Advanced Manufacturing Technology and leads the responsive manufacturing research group at Nottingham University. Research interests include machining and tooling technology, feature-based CAD/CAM systems, generative process planning, integrated planning and scheduling systems, CNC part programming and the design and confguration of manufacturing systems. 